\title{
ENGEVISTA
}

Página da revista: http://www.uff.br/engevista/seer/

\section{Propriedades estruturais e eletrônicas da hidroxiapatita a partir de cálculos de primeiros princípios}

\author{
Welberth Santos Ferreira ${ }^{1}$ \\ Cassandra Maria Ribeiro Costa ${ }^{2}$ \\ Suelen Rocha Botão Ferreira ${ }^{3}$ \\ Sandra Fernanda Nunes ${ }^{4}$
}

Resumo: Neste trabalho foi efetuado um estudo sistemático das propriedades estruturais e eletrônicas da hidroxiapatita (HA), através da teoria funcional de densidade (DFT) usando o código - CASTEP. Os resultados obtidos através da técnica de aproximação GGA - PBE demonstraram em termos das propriedades estruturais uma diferença máxima de $13.4 \%$ entre o valor teórico e experimental, enquanto o volume calculado com estes valores foi $\mathrm{V}=529,58 \AA^{3}$.

Palavras-chave: Biocerâmicas, biomateriais, hidroxiapatita, DFT.

\begin{abstract}
In this work was carried out a systematic study of structural and electronic properties of hydroxyapatite (HA) through the density functional theory (DFT) using the CASTEP code. The calculations were obtained using generalized gradient approximation (GGA) with pseudopotencial Perdew-Burke- Ernzerhof (PBE) and demonstrated which the structural properties presents a maximum difference of $13.4 \%$ between theoretical and experimental values, in the other hand the volume were $529,58 \AA^{3}$.
\end{abstract}

Keywords: Bioceramics, biomaterials, hydroxyapatite, DFT, density functional theory.

\footnotetext{
${ }^{1}$ UEMA - Universidade Estadual do Maranhão

2 UEMA - Universidade Estadual do Maranhão

${ }^{3}$ UEMA - Universidade Estadual do Maranhão

${ }^{4}$ UEMA - Universidade Estadual do Maranhão
} 


\section{Introdução}

O desenvolvimento de biomateriais cerâmicos tem atraído a atenção da comunidade científica nos últimos anos. Desde então os biomateriais vem sendo utilizados em diversos campos de estudos como enxertos, reparação óssea odontológica e da cartilagem articular, por serem seguros, eficazes e biologicamente aceitáveis. Sendo assim, os biomateriais são definidos como biocompatíveis, apresentando propriedades mecânicas, físicas e químicas adequadas para recuperação das diversas funções do organismo humano.

Os primeiros estudos sobre a utilização desses materiais cerâmicos como biomateriais começaram por Albee e seus colaboradores em 1930. Somente a partir dos anos 50 começaram a surgir variados tipos de cerâmicas de fosfato de cálcio (Albbe, 1920; Slepko e Demkov, 2015). Em 1975, Nery e seus colaboradores utilizaram o termo fosfato de cálcio para descrever uma biocerâmica constituída tanto de hidroxiapatita (HA) quanto de $\beta$-fosfato tricálcico, esses materiais são facilmente sintetizados e dependendo de sua esquiometria e nanoestrutura podem apresentar diversas propriedades de interesse na área médica e odontológica (Araujo, 2010).

As biocerâmicas a base de sais de fosfato de cálcio - $\mathrm{Ca} / \mathrm{P}$ vem sendo amplamente utilizada devido ao seu alto grau de biocompatibilidade, que está relacionada com a sua similaridade química, encontrada no tecido ósseo (Davies, 1990). De acordo com Araújo et al., (2007) dentre estes fosfatos, a HA possui grande importância, pois é o que apresenta maior estabilidade termodinâmica próxima da temperatura ambiente.

A HA é o principal constituinte mineral natural encontrado no osso representando de $30 \%$ a $70 \%$ da massa dos ossos e dentes (Costa et al., 2009), sendo sem dúvida a mais estudada e utilizada na medicina. Além disso, a HA é considerada um dos biomateriais mais promissores devido sua biocompatibilidade, bioatividade e osteocondutividade (indicam o caminho para o crescimento ósseo, fazendo que ocorra sobre a superfície ou através dos poros), tornando-a adequada para aplicações biológicas. Embora a estrutura da apatita mineral seja conhecida desde 1930, a estrutura da HA foi realmente esclarecida em 1964, quando as posições atômicas do hidrogênio foram estabelecidas pela difração de raios $-\mathrm{X}$.

Trata-se de um composto que possui estequiometria $\mathrm{Ca}_{10}\left(\mathrm{PO}_{4}\right)_{6}(\mathrm{OH})_{2}$, e cristalografia definidas, cuja razão molar $\mathrm{Ca} / \mathrm{P}$ é igual a 1,67. Sendo assim constituída a partir de uma rede tridimensional de óxido de cálcio e poliedros de fosfato. A sua célula unitária contém uma representação completa do cristal de apatita, consistindo em grupos de $\mathrm{Ca}, \mathrm{PO}_{4}$ e $\mathrm{OH}$ (Ibuki, 2014). A célula unitária da HA é composta por 44 átomos, incluindo 10 átomos de cálcio, 6 tetraedros de $\mathrm{PO}_{4}$ e 2 grupos de $\mathrm{OH}^{-}$todos organizados em estrutura hexagonal (Cardoso, 2014).

A HA consiste de um esqueleto de tetraedros do grupo $\left(\mathrm{PO}_{4}{ }^{3}\right)$ com dois átomos de oxigênio no plano basal e outros dois alinhados com o eixo cristalográfico $c$. Os tetraedros do grupo $\left(\mathrm{PO}_{4}{ }^{3}\right)$ estão distribuídos de tal maneira que dão origem a dois tipos de canais, compostos por paredes de ânions de $\mathrm{O}$, perpendiculares ao plano basal. O primeiro canal é ocupado por íons de $\mathrm{Ca}^{2+}$ (cálcio 1), 
que estão localizados a 0 e $1 \frac{1}{2}$ do parâmetro cristalino. O segundo canal é ocupado por íons de $\mathrm{Ca}^{2+}$ (cálcio 2), localizados a 1/4 e 3/4 do parâmetro cristalino (Gouveia, 2008).

A estrutura da HA permite substituições catiônicas e aniônicas isomorfas com grande facilidade: o $\mathrm{Ca}^{2+}$ pode ser substituído por metais tais como o $\mathrm{Mg}^{2+}, \mathrm{Pb}^{2+}, \mathrm{Cd}^{2+}, \mathrm{Cu}^{2+}, \mathrm{Zn}^{2+}, \mathrm{Sr}^{2+}, \mathrm{Co}^{2+}$, $\mathrm{Fe}^{2+}$, etc.; os grupos $\mathrm{PO}_{4}{ }^{3-}$ podem ser substituídos por carbonatos e vanadatos e, os grupos $\mathrm{OH}^{-}$por carbonatos, flúor e cloro (Costa et al., 2009). Essas substituições podem alterar a cristalinidade, os parâmetros de rede, as dimensões cristalográficas, a estabilidade, a bioatividade, a biocompatibilidade, a solubilidade e as propriedades de adsorção da estrutura da HA sem que ocorra significante mudança na simetria hexagonal da estrutura (Ibuki, 2014).

De acordo com o processo de fabricação, as propriedades da HA apresentam características diferentes e são classificadas em dois tipos: quando sintetizadas em altas temperaturas, apresentam boa cristalinidade e cristais grandes, e quando sintetizadas em baixas temperaturas, apresentam baixa cristalinidade e cristais pequenos como descrito por Costa et al., (2009).

Nesse contexto, a teoria do funcional da densidade (DFT) tem-se revelado uma ferramenta muito importante para obtenção de um conhecimento mais profundo das propriedades eletrônicas e ópticas da HA (Cardoso, 2014).

Neste trabalho, utilizando o código CASTEP, foi efetuado um estudo sistemático das propriedades estruturais e eletrônicas da HA.

\section{Metodologia}

Para obtenção das bandas eletrônicas da HA foram utilizados os dados cristalográficos de Cardoso (2014) (Cardoso, 2014). Os parâmetros de rede obtidos pelo autor foram: $a=b=9,425 \AA \mathrm{e}$ $c=6,884 \AA$, sendo que os ângulos foram: $\alpha=\beta=90^{\circ}$ e $\gamma=120^{\circ}$, e o volume V $=529,58 \AA^{3}$.

O código CASTEP (Segall et al., 2002) foi utilizado para realizar os cálculos via Teoria do Funcional da Densidade (Hohenberg e Kohn, 1964). Foi considerado a aproximação do gradiente generalizado (GGA), aliado ao funcional Perdew - Burke - Ernzerkof (PBE) (Perdew et al., 1996), e pseudopotencial de norma - conservada, levando em consideração a seguinte configuração: hidrogênio $\left(1 \mathrm{~s}^{1}\right)$, fósforo $\left(3 \mathrm{~s}^{2}-3 \mathrm{p}^{3}\right)$, e oxigênio $\left(2 \mathrm{~s}^{2}-2 \mathrm{p}^{4}\right)$. Para os átomos de cálcio foram usadas duas configurações de valência diferentes: Ca2 $\left(4 s^{2}\right)$ e Ca10 $\left(3 s^{2}-3 p^{6}-4 s^{2}\right)$.

Uma amostragem de Monkhorst - Pack (Monkhorst e Pack, 1976) (grid 2 x 2 x 2) da zona de Brillouin foi utilizado para calcular todas as integrais no espaço recíproco, suficiente para garantir a convergência na estrutura eletrônica.

\section{Resultados e discussão}

\subsection{Otimização Geométrica}

Visando obter uma excelente otimização geométrica foram considerados os seguintes limites de convergência para cálculos de autoconsistência: o valor inicial da variação da energia total foi de 
$2.0 \times 10^{-5} \mathrm{eV} / \AA ̊ A, 0.05 \mathrm{eV} / \AA ̊$ para a força máxima sobre cada átomo, $0.01 \mathrm{GPa}$ para a pressão e 0.001 Å para o deslocamento atômico máximo.

O algoritmo de otimização foi o Broyden - Fletcher - Goldfard - Shanno (BFGS), adotado com uma energia de corte igual a $750 \mathrm{eV}$.

Otimizados os parâmetros de redes, ângulos e posições atômicas foi considerado uma energia mínima para a célula unitária de HA. A Fig. 1 mostra a célula unitária da HA.

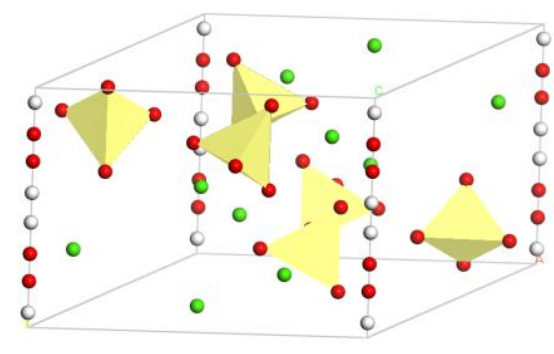

Figura 1. Estrutura cristalina da hidroxiapatita.

Novas técnicas com vistas as crescentes necessidades de alternativas para o reparo e substituição óssea faz-se necessário devido a busca incansável do ser humano pela longevidade e por melhores padrões de vida (Pereira et al., 1994).

A Tabela 1 apresenta a comparação dos parâmetros de redes e volume da célula obtidos através de medidas experimentais realizadas por Cardoso (2014). Em adição, são apresentados os desvios calculados entre os valores experimentais.

Tabela 1. Parâmetros de rede para HA de acordo com abordagem DFT - GGA - BGSH. Também são apresentados os dados experimentais de Cardoso (2014). Onde a, b, e c são os comprimentos e um volume $(\mathrm{V}) \mathrm{em} \mathrm{A}^{3}$. Entre parênteses: também são apresentados os desvios entre os valores experimentais e calculados.

\begin{tabular}{|c|c|c|c|c|}
\hline & $\boldsymbol{A}$ & $\boldsymbol{b}$ & $\boldsymbol{C}$ & $\boldsymbol{V}$ \\
\hline \multicolumn{5}{|c|}{$\mathbf{C a}_{10}\left(\mathbf{P O}_{4}\right)_{\mathbf{6}}(\mathbf{O H})_{2}$} \\
\hline DFT- GGA & $8.162(-13.4 \%)$ & $9.325(-0.13 \%)$ & $6.894(0.22 \%)$ & $529.583(-0.07 \%)$ \\
\hline $\begin{array}{c}\text { Experimento } \\
\text { Cardoso } \\
\text { (2014) }\end{array}$ & 9.425 & 9.425 & 6.884 & 530 \\
\hline
\end{tabular}

A Tabela 2 apresenta uma comparação entre os dados experimentais obtidos por Dourado (2006) e os nossos dados teóricos. 
Tabela 2. Títulos relevantes, comprimentos, distâncias e ângulos de HA. Os dados obtidos em GGA e os resultados experimentais (exp) são representados para comparação (no plano xz).

\begin{tabular}{|c|c|c|}
\hline $\mathrm{Ca}_{10}\left(\mathrm{PO}_{4}\right)(\mathrm{OH})_{2}$ & $\begin{array}{c}\text { Experimento } \\
\text { Dourado (2006) }\end{array}$ & GGA \\
\hline $\mathrm{Ca} 1-\mathrm{O} 1(\AA)$ & 2.41 & $2.41(-0 \%)$ \\
\hline $\mathrm{Ca} 1-\mathrm{O} 2(\AA)$ & 2.45 & $2.44(-0.4 \%)$ \\
\hline $\mathrm{Ca} 1-\mathrm{Ca} 1(\AA)$ & 3.43 & $3.42(-0.2 \%)$ \\
\hline $\mathrm{Ca} 2-\mathrm{O} 2(\AA)$ & 2.36 & $2.34(-0.8 \%)$ \\
\hline $\mathrm{Ca} 2-\mathrm{OH}(\AA)$ & 2.39 & $2.34(-2.1 \%)$ \\
\hline $\mathrm{Ca} 2-\mathrm{O} 3(\AA)$ & 2.51 & $2.37(-5.9 \%)$ \\
\hline $\mathrm{Ca} 2-\mathrm{Ca} 2(\AA)$ & 4.09 & $4.16(-0.2 \%)$ \\
\hline $\mathrm{P}-\mathrm{O} 1(\AA)$ & 1.54 & $1.53(-0.6 \%)$ \\
\hline $\mathrm{P}-\mathrm{O} 2(\AA)$ & 1.54 & $1.55(-0.6 \%)$ \\
\hline $\mathrm{P}-\mathrm{O} 3(\AA)$ & 1.51 & $1.51(-0 \%)$ \\
\hline $\mathrm{O} 2-\mathrm{Ca} 1-\mathrm{O} 2(\mathrm{deg})$ & 75 & $78.255(-4.1 \%)$ \\
\hline $\mathrm{O} 3 \mathrm{~B}-\mathrm{Ca} 2-\mathrm{O} 3(\mathrm{deg})$ & 58 & $61.010(-4.9 \%)$ \\
\hline $\mathrm{O} 3{ }_{\mathrm{A}}-\mathrm{Ca} 2-\mathrm{O} 2(\mathrm{deg})$ & 86 & $81.078(-6.1 \%)$ \\
\hline $\mathrm{O} 3_{\mathrm{B}}-\mathrm{Ca}-\mathrm{O} 2(\mathrm{deg})$ & 75 & $81.078(-7.4 \%)$ \\
\hline $\mathrm{O} 1-\mathrm{P}-\mathrm{O} 2(\mathrm{deg})$ & 111 & $112.109(-0.9 \%)$ \\
\hline $\mathrm{O} 1-\mathrm{P}-\mathrm{O} 3(\mathrm{deg})$ & 108 & $111.427(-3.0 \%)$ \\
\hline $\mathrm{O} 2-\mathrm{P}-\mathrm{O} 3(\mathrm{deg})$ & 111 & $107.356(-3.3 \%)$ \\
\hline
\end{tabular}

\subsection{Densidade de Estados Parcial}

A partir da formação de equipes multidisciplinares entre engenheiros biomédicos, químicos físicos, biólogos e médicos, os biomateriais têm melhorado de maneira significativa o seu desempenho e alcançado os requisitos mínimos para aplicação na prática médica/odontológica (Laurenti, 2011). 
A Fig. 2 apresenta a densidade de estados parcial (PDOS) do nosso sistema em função da energia $(\mathrm{em} \mathrm{eV})$, para as espécies atômicas $\mathrm{Ca}, \mathrm{P}, \mathrm{O}$ e H. A direita são mostradas as contribuições de cada uma das espécies atômicas. As linhas contínuas, tracejadas e pontilhadas correspondem às contribuições $s, p, d e f$, respectivamente. O nível de Fermi é considerado como sendo $0.0 \mathrm{eV}$. A contribuição mais relevante para o átomo de Cálcio $(\mathrm{Ca})$ do orbital $\mathrm{Ca}-3 \mathrm{~s}$ surge com uma variação de energia de - $42.34 \mathrm{eV}$ e - $41 \mathrm{eV}$. Entre os picos - $25.22 \mathrm{eV}$ e $-22.67 \mathrm{eV}$ é observado a contribuição do orbital $\mathrm{P}-3 \mathrm{~s}$ e -25 . eV e, por último, a $-20 \mathrm{eV}$ são do orbital $\mathrm{O}-2 \mathrm{~s}$.
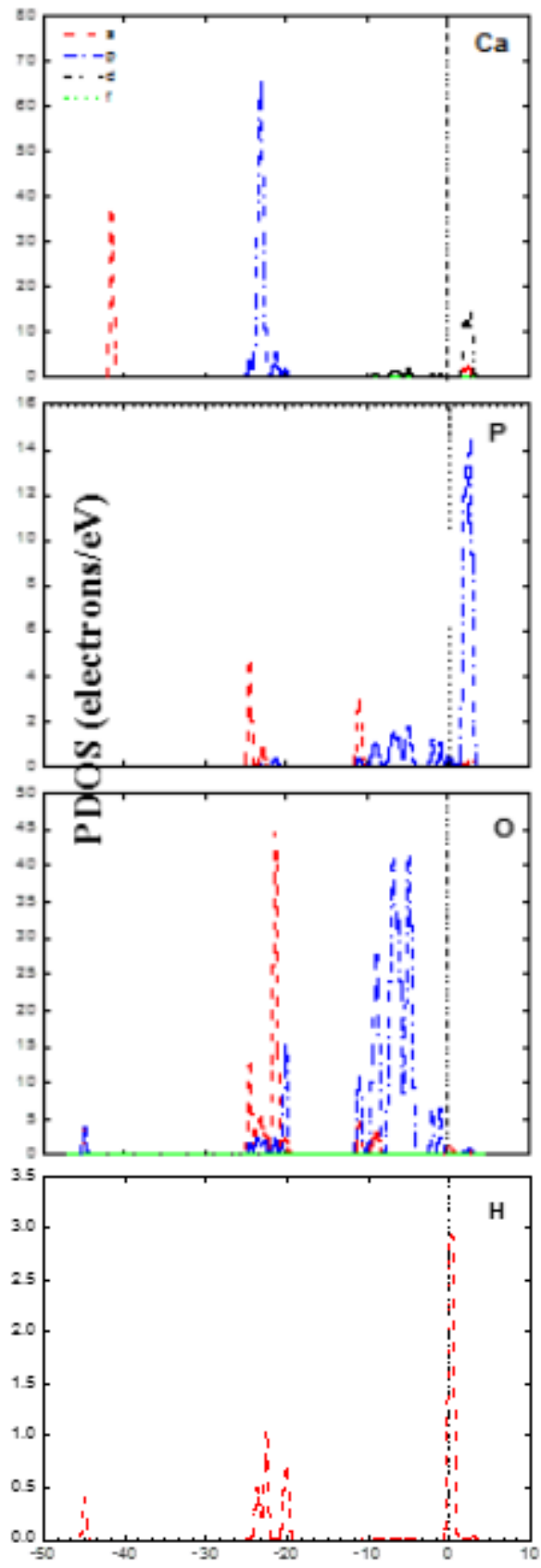

ENERGIA (eV)

Figura 2. Densidade de estado parcial (PDOS para os átomos $\mathrm{Ca}, \mathrm{P}, \mathrm{O}$ e $\mathrm{H}$ calculado com o funcional de troca-correlação GGA - PBE) 
Com a metodologia aplicada neste estudo, a HA revelou-se ser de extrema importância na aplicação biomédica por ser o principal mineral constituinte do osso, sendo necessário avaliar macroscopicamente e histologicamente reparos osteocondriais já cicatrizados como descrito por Ribeiro et al., 2004.

\section{Conclusão}

Em termos das propriedades estruturais, utilizando a aproximação GGA - PBE observouse uma diferença máxima entre o valor teórico e experimental de $13.4 \%$, que corresponde ao parâmetro de rede $a$ do cristal, enquanto o volume calculado com estes valores foi $V=529,58 \AA^{3}$, apresentando boa concordância com trabalhos previamente publicados na literatura (Slepko e Demkov, 2015; Araujo et al., 2010; Cardoso, 2014). Indicando que a HA pode ser utilizada como biomaterial que imita o comportamento ósseo.

A partir dos resultados obtidos, pode-se concluir que a modelagem computacional é uma ferramenta útil, pois através dela é possível analisar a estrutura cristalina da HA.

Utilizando este mecanismo para obtenção de uma nova estrutura que reproduzisse o mínimo de erros dos parâmetros estruturais e do volume para a célula da HA.

\section{Agradecimentos}

Este trabalho foi financiado pela Fundação de Amparo à Pesquisa e ao Desenvolvimento Científico e Tecnológico do Maranhão (FAPEMA), (APP - 00279/10).

\section{Referências}

ALBBE, F. H. 1920. Studies in Boné Growth Triple Calcium Phosphate as Stimulus to Osteogenesis. Annual of Surgery, 71, 32.

ARAÚJO, J. C., SENA, L., BASTOS, I. N. \& SOARES, G. D. 2007. A. Síntese da Hidroxiapatita e Refinamento Estrutural por Difração de Raios-X. Quim. Nova, 30, 1853 - 1859.

ARAUJO, J. C., BASTOS, I. N. \& SOARES, G. D. A. 2010. Determinação Estrutural da Hidroxiapatita por Síntese de Fourier e Método de Rietveld. Engevista, 12, 41-48.

CARDOSO, P. S. 2014. Preparation and Structural Characterization of Hydroxyapatite Thin Films for Biomedical Applications. Dissertação de Doutorado. Faculdade de Ciências da Universidade do Porto.

COSTA, A. C. F. M., LIMA, L. H. M. A., CORDEIRO, V. V., VIANA, K. M. S., SOUZA, C. V. \& LIRA, H. L. 2009. Hidroxiapatita: obtenção, caracterização e aplicações. Revista Eletrônica de Materiais e Processos, 4, 29-38.

DAVIES, J. E. 1990. The bone material interface. University of Toronto Press.

DOURADO, E. R. 2006. Preparação e caracterização de hidroxiapatita nanoestruturada dopada com estrôncio. Dissertação de Mestrado, Centro Brasileiro de Pesquisas Físicas. Rio de Janeiro. 
HOHENBERG, P. \& KOHN, W. 1964. Inhomogeneous Electron Gas. Phys. Rev, 136, B864. IBUKI, F. K. 2014. Síntese e caracterização de nanopartículas de hidroxiapatita funcionalizadas e análise da interação destas com o tecido dentinário. Dissertação de doutorado, Universidade de São Paulo.

GOUVEIA, D. S. 2008. Nanométricos de Hidroxiapatita Sintetizados com Magnésio Utilizando Ultra-Som. Dissertação de Doutorado, Universidade de São Paulo.

LAURENTI, K. C. 2011. Desenvolvimento de um biomaterial composto de poliuretano e microfibra de biovidro 45S5 em gradiente funcional para reparo de cartilagem articular: estudos in vitro e in vivo. Dissertação de Doutorado, Escola de Engenharia de São Carlos da Universidade de São Paulo.

MONKHORST, H. J. \& PACK J. D. 1976. Special points for Brillouin-zone integrations. Phys. Rev. B 13, 5188.

PERDEW, J. P., BURKE, K. \& ERNZERHOF, M. 1996. Generalized Gradient Approximation Made Simple. Phys. Rev. Lett. 77, 3865.

PEREIRA, M. M., CLARK, A. E. \& HENCH, L. L. 1994. Calcium phosphate formation on solgel-derived bioactive glasses in vitro. J Biomed Mater Res., 28, 693-698.

RIBEIRO, A. F., CAMANHO, G. L. \& TAKITA, L. C. 2004. Estudo Macroscópico e histológico de reparos osteocondrais biologicamente aceitáveis. Acta Orthop Bras, 12, 16-24.

SEGALL, M. D., LINDAN, P. J. D., PROBERT, M. J., PICKARD, C. J., HASNIP, P. J., CLARK, S. J. \& PAYNE, M. C. 2002. First-principles simulation: ideas, illustrations and the CASTEP code. J. Phys. Condens. Matter. 14, 2717.

SLEPKO, A. \& DEMKOV, A. A. 2015. Hydroxyapatite: vibracional spectra and monoclinic to hexagonal phase transition. Journal of Applied Physics, 117, 074701. 